\title{
Accuracy of Soft Tissue Balancing in Robotic- Assisted Measured-Resection TKA Using a Robotic Distraction Tool
}

\author{
Jan A. Koenig ${ }^{1}$, Sami Shalhoub ${ }^{2}$, Eric A. Chen ${ }^{1}$, Christopher Plaskos ${ }^{2}$ \\ ${ }^{1}$ NYU Winthrop University Hospital, Long Island, NY, USA \\ ${ }^{2}$ Omnilife Science, Boston, USA
}

\begin{abstract}
Achieving proper soft tissue balance during total knee arthroplasty (TKA) can reduce post- operative instability and stiffness as well as improve patient reported outcomes. The objective of this study was to compare final intra-operative coronal balance throughout the knee range of motion in navigated roboticassisted TKA when performed with quantifiable feedback from a robotic ligament tensioning tool versus with standard trials and navigation measurements alone.

The study included a prospective cohort of 52 patients undergoing roboticassisted TKA using a measured resection technique. The cohort was divided into two sequential groups: a non-sensor-assisted group $(\mathrm{n}=25)$ and a subsequent sensor-assisted group $(\mathrm{n}=27)$. Once bony cuts and soft tissue balancing was performed in the non-sensor cohort, the final tibiofemoral gaps were measured throughout the knee range of motion using a robotic-assisted tensioner with the surgeon blinded to the measurements. For the sensor cohort, the surgeon preformed soft-tissue releases or re-cuts in order to balance the knee using the gap measurement data from the robotic tensioner. The robotic-assisted tensioner was then used to measure the final medial and lateral gap measurements.

The average mediolateral gap difference throughout the range of flexion was $1.9 \pm 0.7 \mathrm{~mm}$ with maximum difference of $7.8 \mathrm{~mm}$ for the non-sensor cohort. The sensor cohort had an average mediolateral difference of $1.5 \pm 0.6 \mathrm{~mm}$ and a maximum difference of $3.8 \mathrm{~mm}$. The difference between the two groups was statistically significant from 60 to 90 degrees of flexion. 38-41\% of knees were balanced to within $1 \mathrm{~mm}$ mediolaterally in the non-sensor group compared to 48$70 \%$ for the sensor group when measured at various flexion angles. $65-76 \%$ of knees were balanced to within $2 \mathrm{~mm}$ for the non-sensor group compared to 78$86 \%$ for the sensor-assisted group. The number of knees requiring subsequent soft tissue releases was similar in each group. Soft tissue balancing with the aid
\end{abstract}


Accuracy of Soft Tissue Balancing in Robotic-Assisted Measured-Resection TKA ... J. Koenig et al.

of a robotic tensioning tool resulted in significantly more accurate soft tissue balance than when using navigation measurements and standard trials alone in this single surgeon study.

\section{Introduction}

Achieving proper soft tissue balance during total knee arthroplasty (TKA) can reduce postoperative instability and stiffness as well as improve patient reported outcomes [1]. Intra-operative assessment of soft tissue balance relies on the surgeons' experience and subjective feel, and thus can result in variable and inconsistent results [2]. Recent developments in sensor and robotic technologies provide surgeons with the ability to quantitatively measure soft tissue loads and tension and gap balance intra-operatively throughout the range of motion of the knee $[3,4]$. This allows the surgeon to optimize soft-tissue releases and bony resections to achieve a balanced knee. The objective of this study was to compare final intra-operative coronal balance throughout the knee range of motion in navigated robotic-assisted TKA when performed with quantifiable feedback from a robotic ligament tensioning tool versus with standard trials and navigation measurements alone.

\section{Methodology}

The study included a prospective cohort of 52 patients undergoing robotic-assisted TKA using a measured resection technique. The cohort was divided into two sequential groups: 1) a first nonsensor assisted group $(\mathrm{n}=25$, age $=67.2 \pm 8.8$, BMI $30.8 \pm 4.4)$ and 2$)$ a sensor-assisted group $(\mathrm{n}=27$, age $=70.4 \pm 7.5$, BMI $=29.8 \pm 4.4$ ). A hybrid measured resection technique was used to determine femoral component alignment for both groups, where femoral rotation was determined using multiple bony landmarks on the femur and accounting for posterior cartilage wear. Implant planning and femoral cuts were preformed using a robotic-assisted system (OmniBotics, Omni, Raynham MA) and a cruciate retaining femoral trial was inserted. The tibia was resected perpendicular to the mechanical axis. Once the surgeon determined that the knee was balanced in the non-sensor cohort, the final tibiofemoral gaps throughout the range of flexion were measured using a robotic-assisted tensioner (OMNI BalanceBot ${ }^{\mathrm{TM}}$ ) with the surgeon blinded to the measurements. The robotic-assisted tensioner allowed for separate medial and lateral gap measurements while a constant distraction force was applied to the joint. For the sensor-cohort, the robotic-assisted tensioner was inserted into the joint following the femoral cuts to measure the tibiofemoral gaps throughout the range of motion. Using the gap measurement feedback, the surgeon performed soft-tissue releases or re-cuts in order to balance the knee. The robotic-assisted tensioner was then used to measure the final medial and lateral gap measurements.

The medial-to-lateral gap difference was calculated for each patient throughout the range of motion in 10 degrees increments. Average and standard deviation for the mediolateral gap difference was calculated for each group and an unpaired t-test was used to determine significant differences between the two subgroups.

\section{Results}

The average mediolateral gap difference throughout the range of flexion was $1.9 \pm 0.7 \mathrm{~mm}$ with a maximum difference of $7.8 \mathrm{~mm}$ for the non-sensor cohort (Figure 2A). The sensor cohort had an 
average mediolateral difference of $1.5 \pm 0.6 \mathrm{~mm}$ and a maximum difference of $3.8 \mathrm{~mm}$ (Figure 2B). The difference between the two groups was statistically significant from $60,70,80$, and 90 degrees of flexion ( $\mathrm{p}=0.045,0.0084,0.0016$, and 0.001 respectively). The percentage of knees balanced to within $1 \mathrm{~mm}$ mediolaterally ranged from $38-41 \%$ for the non-sensor group compared to $48-70 \%$ for the sensor group (Figure 2C-D). When comparing mediolateral balance to within $2 \mathrm{~mm}$, that percentage range increased to $65-76 \%$ for the non-sensor group compared to $78-86 \%$ for the sensorassisted group. The number of knees requiring subsequent soft tissue releases was similar in each group, with $0,1,2$, and 3 releases required in $40 \%, 48 \%, 12 \%$, and $0 \%$ vs $37 \%, 44 \%, 15 \%$, and $4 \%$ in the non-sensor vs sensor-assisted groups respectively.

\section{Discussion}

The robotic-assisted tensioner allows the surgeon to develop and optimize a plan to address the gap imbalance based on quantitative data instead of relying on feel alone. This results in an increase in mediolateral balance throughout the range of flexion when using the robotic-assisted tensioner compared to subjective feel by the surgeon. Similar results have been observed when using a sensor technology that measures medial and lateral force instead of gaps [2, 5]. The difference in balance between the two cohorts was modest in extension and increased with flexion. This may be due to the relative ease in assessing coronal balance by feel in extension and the increase in difficulty in gauging coronal balance with increasing degrees of flexion.

The study was limited in that it was a single surgeon study. In addition, the non-randomized nature of the patient cohorts may have resulted in imbalances in prognostic factors between the two groups. Blinding the surgeon to the treatment cohorts in this type of prospective cohort study is not feasible, and may also contribute to surgeon bias.

In this pilot study, soft tissue balancing with the aid of a robotic tensioning tool resulted in significantly more accurate soft tissue balance than when using navigation measurements and standard trials alone. This was especially true with deeper degrees of knee flexion, where statistically significant differences in coronal balance became apparent between the two cohorts. As computer assisted and navigation guided TKA technology continues to advance, our ability to quantifiably evaluate intra-operative ligamentous balance will continue to improve. Further studies are necessary in order to evaluate the impact of such fine tuning of TKA coronal balance and its impact on patient outcomes.

A

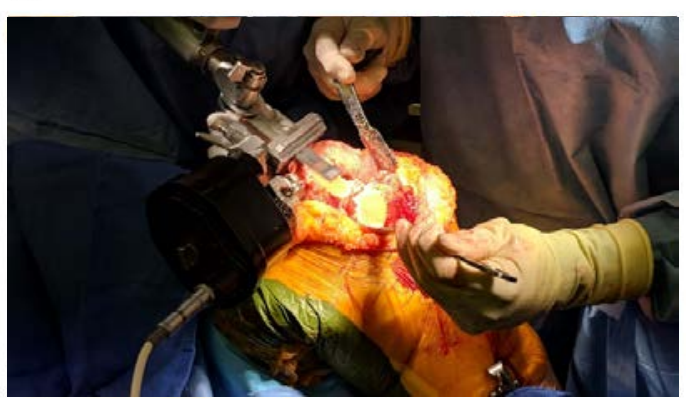

B

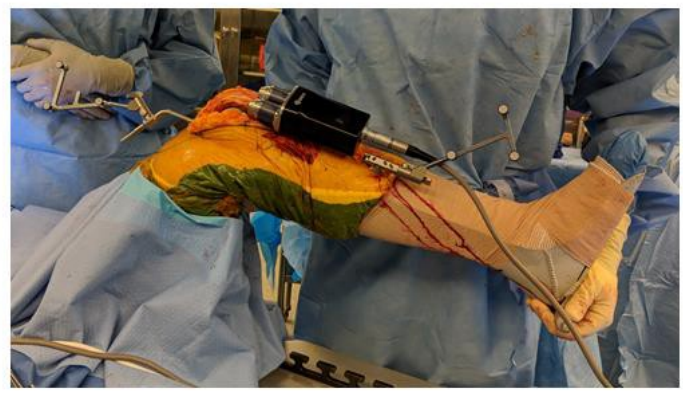

Figure 1: (A) Resection of the femur using the OMNIbot and (B) acquiring the final tibiofemoral gap using the BalanceBot 


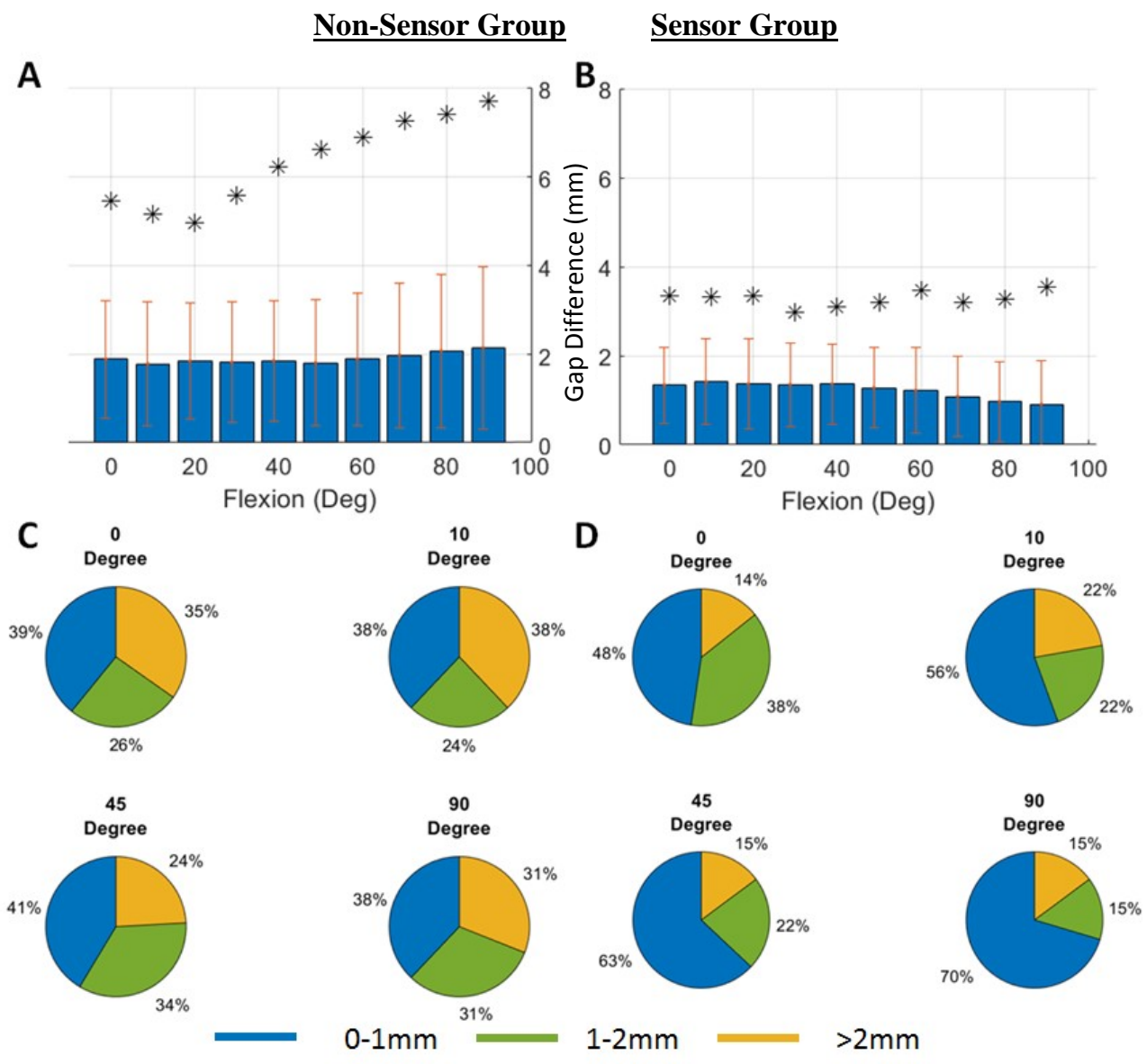

Figure 2: Difference in mediolateral lateral balance through the flexion range for (A) non-sensor cohort and (B) sensor cohort with * representing the maximum medial-to-lateral difference. (C) and (D) represent the percentage of knee balanced within 0-1mm (blue), 1$2 \mathrm{~mm}$ (green), $>2 \mathrm{~mm}$ (orange) for (C) the non-sensor and (D) sensor cohort. 


\section{Reference}

1. Gustke KA, Golladay GJ, Roche MW, Jerry GJ, Elson LC, Anderson CR. Increased satisfaction after total knee replacement using sensor-guided technology. Bone Joint J 96B(10): 1333, 2014

2. Elmallah RK, Mistry JB, Cherian JJ, Chughtai M, Bhave A, Roche MW, Mont MA. Can We Really "Feel" a Balanced Total Knee Arthroplasty? J Arthroplasty 31(9 Suppl): 102, 2016

3. Meere PA, Schneider SM, Walker PS. Accuracy of Balancing at Total Knee Surgery Using an Instrumented Tibial Trial. J Arthroplasty 31(9): 1938, 2016

4. Shalhoub S, Moschetti WE, Dabuzhsky L, Jevsevar DS, Keggi JM, Plaskos C. Laxity Profiles in the Native and Replaced Knee-Application to Robotic-Assisted Gap-Balancing Total Knee Arthroplasty. J Arthroplasty 33(9): 3043, 2018

5. Cho KJ, Seon JK, Jang WY, Park CG, Song EK. Objective quantification of ligament balancing using VERASENSE in measured resection and modified gap balance total knee arthroplasty. BMC Musculoskelet Disord 19(1): 266, 2018 